\title{
Mechanisms of Spontaneous Resolution of Rat Liver Fibrosis Hepatic Stellate Cell Apoptosis and Reduced Hepatic Expression of Metalloproteinase Inhibitors
}

\author{
J.P. Iredale, R.C. Benyon, J. Pickering, M. McCullen, M. Northrop, S. Pawley, C. Hovell, and M.J.P. Arthur
}

University Medicine, University of Southampton, Hampshire SO16 6YD, United Kingdom

\begin{abstract}
Liver fibrosis results from the excessive secretion of matrix proteins by hepatic stellate cells (HSC), which proliferate during fibrotic liver injury. We have studied a model of spontaneous recovery from liver fibrosis to determine the biological mechanisms mediating resolution.

Livers were harvested from rats at $0,3,7$, and $28 \mathrm{~d}$ of spontaneous recovery from liver fibrosis induced by 4 wk of twice weekly intraperitoneal injections with $\mathrm{CCl}_{4}$. Hydroxyproline analysis and histology of liver sections indicated that the advanced septal fibrosis observed at time $\mathbf{0}$ (peak fibrosis) was remodeled over $28 \mathrm{~d}$ of recovery to levels close to control (untreated liver). $\alpha$-Smooth muscle actin staining of liver sections demonstrated a 12-fold reduction in the number of activated HSC over the same time period with evidence of HSC apoptosis. Ribonuclease protection analysis of liver RNA extracted at each recovery time point demonstrated a rapid decrease in expression of the collagenase inhibitors TIMP-1 and TIMP-2, whereas collagenase mRNA expression remained at levels comparable to peak fibrosis. Collagenase activity in liver homogenates increased through recovery.

We suggest that apoptosis of activated HSC may vitally contribute to resolution of fibrosis by acting as a mechanism for removing the cell population responsible for both producing fibrotic neomatrix and protecting this matrix from degradation via their production of TIMPs. (J. Clin. Invest. 1998. 102:538-549.) Key words: liver fibrosis • apoptosis • hepatic stellate cells • TIMP • collagenase
\end{abstract}

\section{Introduction}

Liver fibrosis is characterized by increased deposition and altered composition of extracellular matrix, such that there is an excess of collagens I, III, and IV (1). When advanced, the liver architecture is distorted by dense bands of collagens that link vascular structures and surround islands of regenerating parenchymal cells; these changes are characteristic of cirrhosis. Advanced fibrosis and cirrhosis are generally considered to be

Address correspondence to J.P. Iredale, University Medicine, Level D, South Block, Southampton General Hospital, Tremona Road, Southampton, Hampshire SO16 6YD, UK. Phone: 44-1703-796883; FAX: 44-1703-794154; E-mail: bct@soton.ac.uk

Received for publication 24 June 1997 and accepted in revised form 22 May 1998.

J. Clin. Invest.

(C) The American Society for Clinical Investigation, Inc. 0021-9738/98/08/0538/12 \$2.00

Volume 102, Number 3, August 1998, 538-549

http://www.jci.org irreversible conditions even after removal of the injurious agent. Moreover, the development of cirrhosis is characterized by a diminution of liver collagenase activity, implying that the capacity of the diseased liver to remodel the fibrotic matrix is critically reduced. However, data from the histological assessment of biopsy tissue from patients with liver fibrosis, complicating chronic viral infection, haemochromatosis, and Wilson's disease, who have been successfully treated, and from animal models of fibrosis indicate that recovery with remodeling of the excess collagens is possible (2-4). These data suggest that even in advanced fibrosis a capacity for matrix remodeling exists and that by establishing and analyzing a model of spontaneous recovery from liver fibrosis, key insights into the mechanism of the fibrotic process may be gained. Moreover, such insights may highlight potential avenues on which future therapeutic strategies can be based.

A wealth of evidence now indicates that hepatic stellate cells (HSC, ${ }^{1}$ lipocytes, fat-storing, or Ito cells) are central to the process of fibrosis as the major source of fibrillar and nonfibrillar matrix proteins (1). Quiescent HSC synthesize low levels of matrix proteins, but, as a result of injury, HSCs proliferate and transform to a myofibroblast-like phenotype, a process termed activation (1). Activated HSCs express $\alpha$-smooth muscle actin ( $\alpha$-SMA) and procollagen-I (1) and are known to be the major source of collagens and other matrix proteins that are deposited in fibrosis. Accumulation of matrix, therefore, occurs as a consequence of both an increase in the numbers of HSCs, in addition to their increased synthesis and secretion of matrix proteins when in the activated phenotype. Resolution of liver fibrosis could be associated with a reversal of activated HSCs to a quiescent phenotype or by a change in the balance of cell death over proliferation resulting in a net loss of activated HSCs. An increasing body of evidence suggests that a major mechanism mediating the loss of cells that are redundant or unwanted after growth, development, or a pathological process is "programmed cell death" or apoptosis (5-8). We have, therefore, examined an experimental model of fibrosis through a progressive and recovery phase to determine whether activated HSCs undergo apoptosis during spontaneous resolution of fibrosis in vivo.

The loss of activated HSCs is not in itself sufficient to allow a remodeling of the existing excess collagens. For this to occur, matrix degradation must be upregulated. The key enzyme in the degradation of interstitial collagens I and III is interstitial collagenase (MMP-1). No true rat homologue of this enzyme has been described, instead, degradation of collagen I and III in the rat appears to be mediated by a further collagenase,

1. Abbreviations used in this paper: $\alpha \mathrm{SMA}, \alpha$-smooth muscle actin; HSC, hepatic stellate cell; MMP, matrix-degrading metalloproteinase; RPA, ribonuclease protection assay; TBS, Tris-buffered saline; TIMP, tissue inhibitor of metalloproteinase; TUNEL, terminal deoxynucleotidyl transferase UTP nick end labeling. 
MMP-13 (9). We and others have demonstrated that HSCs are capable of expressing a variety of matrix-degrading metalloproteinases (MMPs), including interstitial collagenase in the human and MMP-13 in the rat (10-15).

We have reported that expression of MMP-1/MMP-13 mRNA remains unchanged during the development of fibrosis, which raises an apparent paradox as collagenolytic activity increases initially, but then falls once advanced fibrosis/cirrhosis occurs in homogenates of fibrotic liver in animal models (16-18). One explanation for these observations is that MMP-1/MMP-13 activity is blocked by the tissue inhibitors of metalloproteinase (TIMPs) 1 and 2, which are expressed by HSC during activation in rat and human models of liver fibrosis $(10,15,19-24)$. We have, therefore, examined the model of spontaneous recovery from established liver fibrosis to further study the possible role of MMP-1/MMP-13 and TIMPs 1 and 2 in the injury-fibrosis-recovery sequence.

\section{Methods}

Progressive fibrosis and fibrosis recovery model. Male Sprague Dawley rats (Indianapolis, IN) weighing 200-300 g with free access to standard chow and water were injected intraperitoneally with $0.2 \mathrm{ml} /$ $100 \mathrm{~g}$ sterile $\mathrm{CCl}_{4}$ in a 1:1 ratio with olive oil twice weekly. For the fibrosis recovery model, this treatment was given for a total of $2 \mathrm{wk}$, after which the dose was reduced to $0.1 \mathrm{ml} / 100 \mathrm{~g}$ for a further $2 \mathrm{wk}$ of treatment. The animals were injected under isofluorane anaesthesia in accordance with Home Office guidelines. 12 animals treated with $\mathrm{CCl}_{4}$ for $4 \mathrm{wk}$ were subdivided into four time points of recovery, at which three animals were killed. Because we intended to study recovery from steady state fibrosis, the first study time point was $3 \mathrm{~d}$ after the final injection of $\mathrm{CCl}_{4}$. We have demonstrated, in previous studies, that the peak effect of acute $\mathrm{CCl}_{4}$ toxicity and the period for maximal hepatocyte apoptosis has passed by $72 \mathrm{~h}$ after an injection (our unpublished observation). Three rats were killed at week 4 (peak fibrosis, $3 \mathrm{~d}$ after the last injection of $\mathrm{CCl}_{4}$ treatment), and then at $4 \mathrm{wk}$ plus 3,7 , and $28 \mathrm{~d}$ of recovery. During the recovery time period, they received no form of treatment (i.e., the recovery was spontaneous). Studies were also performed on liver during progressive fibrosis. Two rats were killed at each of $6,24,48$, and $72 \mathrm{~h}$ after a single dose of $\mathrm{CCl}_{4}$, and livers were also harvested from three rats that had been untreated with $\mathrm{CCl}_{4}$ as normal controls. After harvesting, one lobe from each liver was fixed in buffered formalin for histology of liver sections after staining with hematoxylin and eosin, sirius red, and reticulum stains. The remainder of the liver was snap-frozen in liquid nitrogen in preparation for RNA extraction and biochemical analysis.

Quantization of activated and total HSC during progressive fibrosis and spontaneous recovery from fibrosis. Paraffin-embedded sections of each rat liver at each time point studied during progressive fibrosis and spontaneous recovery from established fibrosis were deparaffinized with xylene and subjected to antigen retrieval by pretreating for $25 \mathrm{~min}$ in a microwave $(800 \mathrm{~W})$ at medium power in $0.01 \mathrm{M}$ citrate buffer ( $\mathrm{pH} \mathrm{6)}$. Specimens were then incubated at room temperature sequentially in 5\% mouse serum (in PBS) for 10 min, FITCconjugated monoclonal anti- $\alpha$-SMA (diluted 1:500 in PBS; Sigma Chemical Co., Poole, UK) with three washes in PBS between each incubation, and then mounted in $15 \mu \mathrm{l}$ of "Propidium iodide/Antifade" (Appligene Oncor, Gaithersburg, MD). Afterwards, each specimen was viewed under fluorescence microscopy and the number of $\alpha$-SMA positive cells counted in 30 random high power fields $(\times 40)$ by a blinded observer. To obtain a photographic record of $\alpha$-SMA positive cells, sections were stained as above, but the fluoresceinlabeled primary antibody was detected by incubation with peroxidase-conjugated anti-fluorescein antibody for $30 \mathrm{~min}$ at room temperature. The sections were then washed in Tris-buffered saline (TBS) and exposed to freshly prepared diaminobenzidine (Sigma Chemical
Co.) for $10 \mathrm{~min}$ at room temperature. Sections were then counterstained in Harris' hematoxylin and dehydrated before mounting. Desmin immunostaining (as a marker of activated and quiescent HSCs [25]) was performed on deparaffinized sections of each rat liver. Slides were treated with an inhibitor of endogenous peroxidase $\left(0.5 \% \mathrm{H}_{2} \mathrm{O}_{2}\right)$, and then washed in TBS. After this step and between each subsequent stage, specimens were washed in TBS. Specimens were then blocked for $10 \mathrm{~min}$ in swine serum before being stained with rabbit anti-desmin (Dako Patts, UK) (1:500 in 1\% BSA in TBS) applied to the sections for $18-24 \mathrm{~h}$ at $4^{\circ} \mathrm{C}$ with appropriate nonimmune IgG controls. A biotinylated swine anti-rabbit $\operatorname{IgG}(1: 200)$ was applied for $30 \mathrm{~min}$ at room temperature. The antibody was then detected by applying streptavidin-biotin peroxidase complexes for 30 min. The peroxidase label was demonstrated using diaminobenzidine for $10 \mathrm{~min}$, after which the slides were counterstained with Harris' hematoxylin dehydrated and mounted. Afterwards, each specimen was viewed by standard microscopy and the number of desmin positive cells counted in 30 random high power fields $(\times 40)$ by a blinded observer.

Determination of numbers of nonparenchymal cells undergoing apoptosis and demonstration of activated HSC apoptosis during spontaneous recovery from liver fibrosis. Sections of each rat liver from each time point studied during progressing and spontaneous recovery from fibrosis were deparaffinized, as described above. Afterwards, a duplicate of each section was stained with propidium iodide, as described by Baker et al. (26), and fragmented DNA was detected in apoptotic cells by the terminal deoxynucleotidyl transferase UTP nick end labeling (TUNEL) technique using a method modified from that described by Gavrieli et al. (27), using the Boehringer TUNELstaining kit (Lewes, UK) according to the manufacturer's instructions. Each slide was then analyzed by a blinded observer who counted the number of nonparenchymal apoptotic figures/bodies present in 30 random high power $(\times 40)$ fields from each specimen. Apoptotic bodies in a distribution compatible with parenchymal cells (hepatocytes) were not counted. To confirm that the apoptotic bodies observed in a nonparenchymal distribution were activated HSCs, representative slides at peak fibrosis were dual stained. First, using the TUNEL technique (as described above), TUNEL positive nuclei were identified by 3-amino-9-ethylcarbazole (Sigma Chemical Co.) staining (red). Then, second, by immunostaining for $\alpha$-SMA (Sigma Chemical Co.), with an alkaline phosphatase-conjugated secondary antibody detected by fast blue with appropriate negative controls.

Hepatic cell extraction and culture. HSCs were extracted from normal rat liver by sequential in situ perfusion with collagenase and pronase followed by density gradient centrifugation, and cultured as described previously (28). Cells were seeded at $1.3 \times 10^{6} / \mathrm{ml}$ on uncoated tissue culture plastic for 7-10 d until a confluent monolayer of myofibroblastic cells were formed. Highly activated cells were obtained by serial passage of cells (second to ninth passage). HSCs were cultured in DME (GIBCO BRL, Paisley, Scotland) supplemented with $16 \%$ FCS and antibiotics. The culture medium was changed every $48 \mathrm{~h}$.

TUNEL, Giemsa, and propidium iodide staining of cultured HSCs. HSCs in primary culture and after passage, and cytospin preparations of detached cells within the culture supernatant were fixed in PBS with $1 \%$ paraformaldehyde. Oligonucleosomal fragmentation of DNA was demonstrated by labeling of $3^{\prime}-\mathrm{OH}$ DNA ends generated during apoptosis by enzymatic addition of digoxygenin-labeled dUTP using terminal deoxynucleotidyl transferase (the so-called TUNEL reaction), using the Boehringer TUNEL Staining Kit according to the manufacturer's instructions. Adherent HSCs in culture after passage, and cytospin preparations of detached cells, were prepared and fixed as above before being stained with Giemsa. For propidium iodide staining, HSCs in primary culture and after passage were fixed and then incubated with proteinase K and RNAase before being stained with propidium iodide as described by Baker et al. (26).

Induction of apoptosis in cultured HSCs. Activated passaged and primary cultures of HSCs were washed three times in serum-free 
DME and then cultured in serum-free DME with no other supplements. In each experiment, parallel wells were returned to media containing 16\% FCS. At 3 and $6 \mathrm{~h}$ after media change, the numbers of apoptotic cells condensing and rounding up were determined in the tissue culture plate monolayer and the media in three $10 \times$ fields in duplicate wells, after acridine orange staining as described by Baker et al. (26).

Measurement of liver hydroxyproline. An $\sim 200$-g segment of snap-frozen liver from each animal of each time point was weighed, hydrolyzed in $\mathrm{HCl}$, and analyzed for total hydroxyproline content, according to the method of Bergman et al. (29), with minor modifications.

RNA extraction. Total RNA was extracted from snap-frozen rat liver from each animal at each time point during recovery from fibrosis and from three normal (untreated) rat livers by homogenization in guanidinium isothiocyanate and purified by the method of Chomczynski and Saachi (30) using acid phenol chloroform. Before application to ribonuclease protection assay, a 4- $\mu$ g aliquot of RNA from each sample was subjected to agarose gel electrophoresis, and stained with ethidium bromide to verify equal sample RNA loading and integrity.

Ribonuclease protection assays (RPAs). The cDNAs for rat TIMP-1, TIMP-2, MMP-13 (the kind gift of N. Partridge and C.
Quinn, Pediatric Research Unit, St. Louis University Medical School, St. Louis, MO), and rat procollagen-I (the kind gift of J. Maher, Liver Center Lab, San Francisco, CA) were subcloned into pGEM plasmids and radiolabeled antisense riboprobes were produced by in vitro transcription in the presence of $\left.{ }^{32} \mathrm{P}\right] \mathrm{UTP}$ (ICN Flow, Thame, Oxon, UK) as described $(10,15)$. RPAs were performed on aliquots of $20-50 \mu \mathrm{g}$ of total liver RNA, using a commercial kit as described (Promega, Southampton, UK) $(10,15)$.

After autoradiography, the density of the major protected fragments in each RPA were quantified by scanning densitometry. The mean density of the major protected fragment in each group at each time point during recovery are expressed relative to 4-wk-value, peak fibrosis (time 0 ) liver that has been assigned the value of $100 \%$. In further experiments, the expression of each mRNA in normal (untreated liver), relative to peak fibrosis was determined.

Quantification of collagenase in whole liver homogenates. Snapfrozen liver samples from time 0 , and 3, 7, and $14 \mathrm{~d}$ of recovery from fibrosis and samples of normal liver $(n=2$ at each time point) were homogenized on ice in sample buffer $(50 \mathrm{mM}$ Tris, $\mathrm{pH} 7.6,0.25 \%$ Triton $\mathrm{X}-100,0.15 \mathrm{M} \mathrm{NaCl}, 10 \mathrm{mM} \mathrm{CaCl}$ ) containing serine- and thiolprotease inhibitors (PMSF $0.1 \mathrm{mM}$, leupeptin $10 \mu \mathrm{M}$, pepstatin A $10 \mu \mathrm{M}$, aprotinin $25 \mu \mathrm{g} / \mathrm{ml}$, iodoacetamide $0.1 \mathrm{mM}$ ). The homogenates were then centrifuged at $4^{\circ} \mathrm{C}$ at $14,000 \mathrm{~g}$ for $30 \mathrm{~min}$ to remove
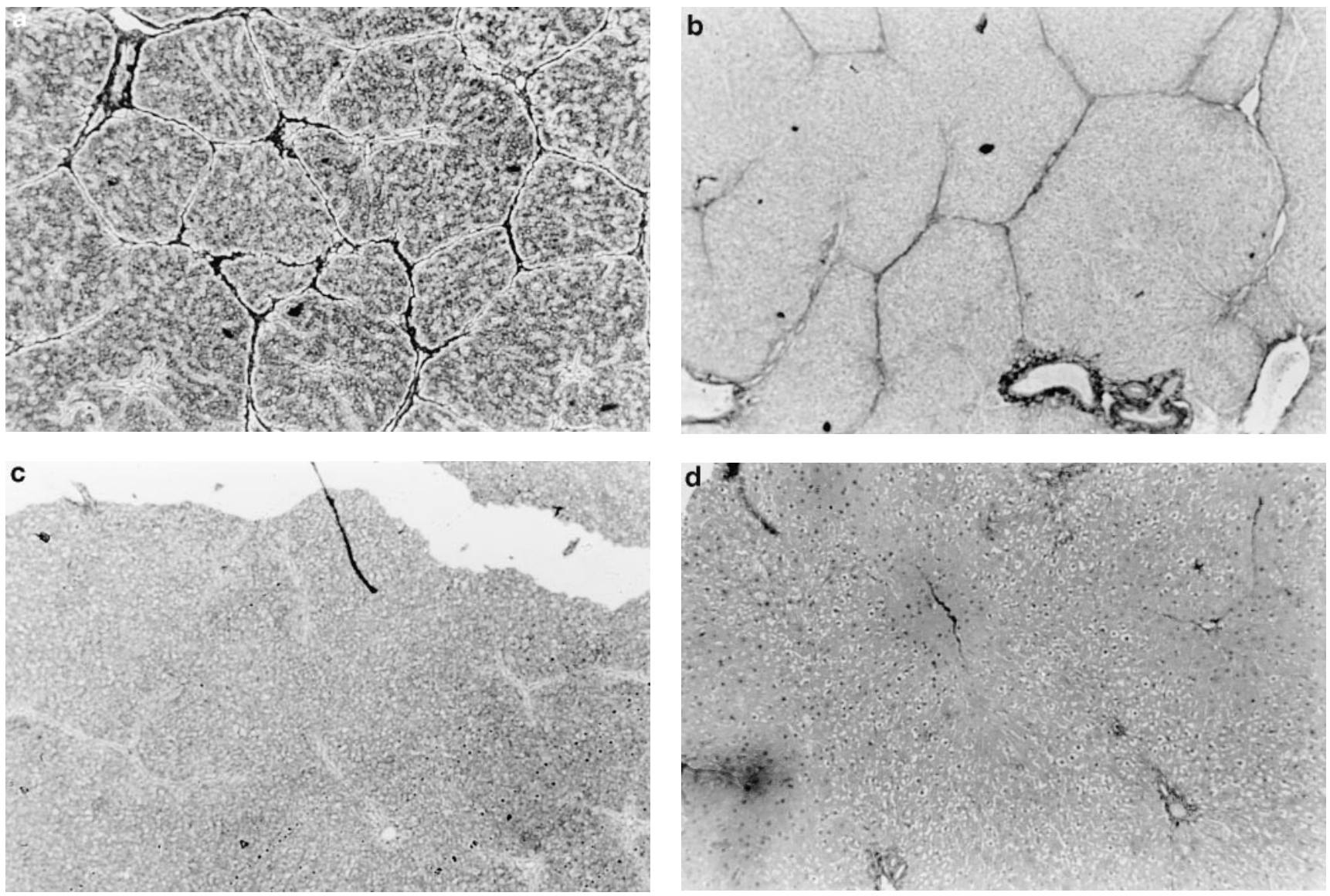

Figure 1. Histological analysis of spontaneous recovery from $\mathrm{CCl}_{4}$ induced liver fibrosis. Liver fibrosis was induced by $\mathrm{IP} \mathrm{CCl}_{4}$ as described in Methods and the extent and distribution of fibrosis assessed by histological analysis of formalin-fixed tissue which had been stained with reticulin and sirius red. At $4 \mathrm{wk}$ (peak fibrosis, $3 \mathrm{~d}$ after final $\mathrm{CCl}_{4}$ injection), there was an obvious nodular appearance with deposition of fibrosis septae, which were continuous and extended between vascular structures ( $a$, representative reticulin-stained section and $b$, representative section stained with sirius red). There was mild fatty change and hepatocyte vacuolation. By day 7 of recovery, the liver appeared more normal histologically with fewer nodules. The fibrous septae were less well defined and discontinuous with clear evidence of collagen matrix degradation. By $28 \mathrm{~d}$, the fibrous septae had largely resolved and only occasional short fibril fragments could be visualized (c, representative reticulin-stained section and $d$, representative section stained with sirius red). Each illustrated micrograph is representative of all three livers studied at the relevant time point. All micrographs $\times 25$ magnification. 
cell debris and protein aggregates. Homogenates were then analyzed in duplicate for interstitial collagenase activity using ${ }^{14} \mathrm{C}$-labeled rat type I collagen as a substrate as previously described (21). Appropriate negative and positive (bacterial collagenase) controls were analyzed in parallel, and the results expressed as cpm of degraded collagen per $\mathrm{mg}$ of protein, determined by optical density at $280 \mathrm{~nm}$ in comparison to serum albumin standards.

Unless otherwise stated, all results are expressed as mean \pm SD for the numbers of independent studies $(n)$ shown.

\section{Results}

Histology of progressive fibrotic liver injury. Histological analysis of the livers harvested at $6 \mathrm{~h}$ after $\mathrm{CCl}_{4}$ demonstrated features that have been described previously, including perivenular ballooning degeneration of hepatocytes; during 24-72 h there was an increase in the inflammatory infiltrate in the necrotic areas and by $1 \mathrm{wk}$ there was evidence of early fibrosis with increased numbers of cells with a fibroblast-like morphology (not shown) in each section.

Histology of spontaneous recovery from carbon tetrachloride-induced liver injury. Histological analysis confirmed that the mature collagen fibrils bridging vascular structures present at week 4 fibrosis become remodeled during the recovery period. At peak fibrosis ( $4 \mathrm{wk}, 3 \mathrm{~d}$ after final $\mathrm{CCl}_{4}$ injection), there was obvious nodular fibrosis with deposition of well delineated fibrous septae, which were continuous and extended throughout each section, illustrated in Fig. 1, $a$ and $b$. Mild fatty change was observed and there was vacuolation of hepatocytes. An increased turnover of hepatocytes was indicated by the presence of mitotic figures. By day 7, the liver appeared to be more normal histologically, the fibrous septae were less well defined, and discontinuous and fewer nodules of regenerative parenchyma were noted. By day 28 , the fibrous septae had largely resolved and only occasional short fibril fragments could be visualized (Fig. 1, $c$ and $d$ ).

Hydroxyproline levels during spontaneous recovery from fibrosis. Determination of tissue hydroxyproline content confirmed the histological studies, showing a net reduction in total collagen levels during the $28 \mathrm{~d}$ of recovery from fibrosis (Fig. 2). Total liver hydroxyproline levels at peak fibrosis were $2.4 \pm 1.4 \mathrm{mmol}$ hydroxyproline/g wet weight and decreased to $0.63 \pm 0.15 \mathrm{mmol}$ hydroxyproline $/ \mathrm{g}$ wet weight at $7 \mathrm{~d}$ and $0.78 \pm 0.45 \mathrm{mmol}$ hydroxyproline $/ \mathrm{g}$ wet weight at $28 \mathrm{~d}$ of recovery, a level comparable with normal untreated liver (Fig. 2).

Spontaneous recovery from established hepatic fibrosis is accompanied by diminution in the numbers of activated HSC. Sections of liver stained with hematoxylin and eosin at peak fibrosis (4 wk) demonstrated large numbers of nonparenchymal cells with a morphology consistent with activated myofibroblast-like HSCs clustered around the fibrotic septae. With recovery from fibrosis, the number of these cells was demonstrated to diminish rapidly over $7 \mathrm{~d}$, and by $28 \mathrm{~d}$, only occasional clusters were visible. This diminution in cell number was not accompanied by morphological evidence of necrosis or inflammation. Because activation of HSC is associated with expression of $\alpha$-SMA (in the majority of cells) (25), immunostaining for this protein was used to detect and quantify activated HSC in each specimen of liver during the development of fibrosis and spontaneous recovery from fibrosis. In normal (untreated) liver, there were $3 \pm 3 \alpha$-SMA positive cells per 30 high power fields $(n=3)$. During the development

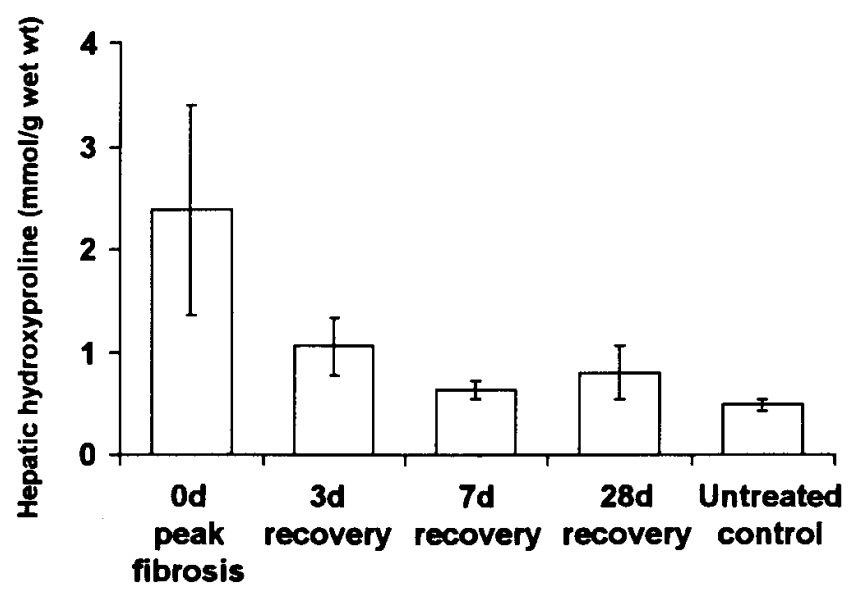

Figure 2. Collagen content of liver during recovery determined by hydroxyproline analysis. Hydroxyproline levels in snap-frozen samples of each liver during spontaneous recovery from $\mathrm{CCl}_{4}$-induced fibrosis were determined as described in Methods. Representative of two independent hydroxyproline analyses of each liver sample analyzed in triplicate ( $n=3$ for each time point).

model of fibrosis, at $6 \mathrm{~h}$ after $\mathrm{CCl}_{4}, 26 \pm 15 \alpha$-SMA positive cells were observed per 30 high power fields $(\times 40)$, the mean number of $\alpha$-SMA positive cells then rose to $195 \pm 21$ per 30 high power fields at $72 \mathrm{~h}$ after $\mathrm{CCl}_{4}$ injection (Fig. $\left.3 a\right)(n=2$ at each time point). At 4 wk (peak fibrosis), 2,850 $\pm 317 \alpha$-SMA positive cells were observed per 30 high power fields $(\times 40)$ $(n=3)$. With progressive recovery from fibrosis, the mean number of $\alpha$-SMA positive cells per 30 high power fields fell progressively from $1,238 \pm 338$ at day 3 falling to $236 \pm 45$ at $28 \mathrm{~d}$ of recovery ( $n=3$ at each time point) (Fig. $3 a$ ).

The distribution of $\alpha$-SMA positive cells within the liver sections was very clearly defined during spontaneous recovery from fibrosis. From peak fibrosis to $7 \mathrm{~d}, \alpha$-SMA positive cells were observed in clusters around fibrotic septae or the residue of fibrotic septae (Fig. 3 b). By 28 d, only scattered $\alpha$-SMA positive cells were observed (Fig. $3 c$ ); these were identified in a perisinusoidal distribution and in association with the residue of fibrotic septae.

Desmin positive cells were quantified during the progressive fibrosis model and found to increase in numbers from $8 \pm 5$ per 30 high power fields in normal liver $(n=3)$ to $68 \pm 20$ per 30 high power fields $(n=2)$ at $6 \mathrm{~h}$ after $\mathrm{CCl}_{4}$ injection, and then to $109 \pm 8.5$ and $120 \pm 115$ per 30 high power fields at 24 and $72 \mathrm{~h}$, respectively, after injection $(n=2$ at each time point). Desmin positive cells were observed predominantly in clusters around fibrotic septae in the 4-wk (peak fibrosis) sections when there were $1,417 \pm 152$ per 30 high power fields. Thereafter, the numbers of desmin positive cells observed fell progressively to $760 \pm 209$ per 30 high power fields after $3 \mathrm{~d}$ of recovery, $405 \pm 109$ after $7 \mathrm{~d}$ of recovery, and $259 \pm 85$ after $28 \mathrm{~d}$ of recovery ( $n=3$ at each time point).

Spontaneous recovery from established fibrosis is accompanied by evidence of nonparenchymal cell apoptosis. After dual staining with TUNEL and $\alpha$-SMA, apoptotic TUNEL positive nuclei in a distribution associated with the collagen fibrils were demonstrated to be within $\alpha$-SMA positive cells, i.e., activated 

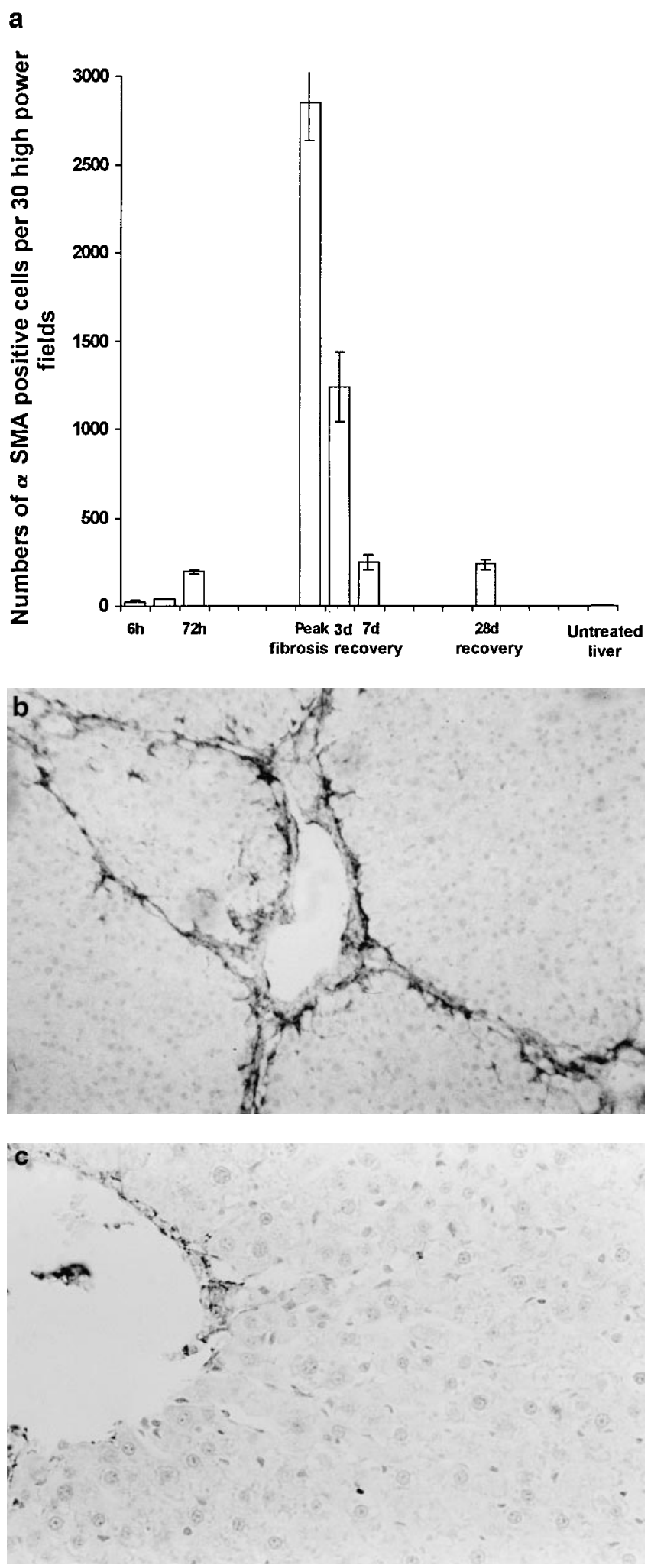

Figure 3. Numbers of $\alpha$-SMA positive activated hepatic stellate cells during progressive experimental fibrosis and spontaneous recovery from liver fibrosis. Liver sections at 6,24 , and $72 \mathrm{~h}$ after a single injection of $\mathrm{CCl}_{4}(n=2$ at each time point) and at $4 \mathrm{wk}$ (peak fibrosis, $3 \mathrm{~d}$ after final $\mathrm{CCl}_{4}$ injection) and after 3, 7, and $28 \mathrm{~d}$ of spontaneous recovery and normal untreated liver ( $n=3$ at each time point) were stained for $\alpha$-SMA (as described in Methods). The number of positive cells stained were counted in 30 random high power fields for
HSC, indicating that HSC apoptosis was taking place during spontaneous recovery from established fibrosis (Fig. 4, $a, b$, and $c$ ).

Apoptosis in nonparenchymal cells, in association with the collagen fibrils and in a nonparenchymal distribution, were quantified by TUNEL and propidium iodide staining. During the progressive model of fibrosis, the number of apoptotic figures in a nonparenchymal distribution detected by propidium iodide staining was found to increase from $7.5 \pm 0.76 \mathrm{~h}$ after $\mathrm{CCl}_{4}$ to $12 \pm 2.872 \mathrm{~h}$ after $\mathrm{CCl}_{4}(n=2$ at each time point) for 30 high power fields $(\times 40)$ (Fig. $5 a$ ). A similar pattern was observed with TUNEL staining. TUNEL positive apoptotic figures in a nonparenchymal distribution numbered $2 \pm 1$ in normal liver $(n=3)$ and $1 \pm 1.4$ at $6 \mathrm{~h}$ after $\mathrm{CCl}_{4}$ before rising to $3 \pm 1$ at $72 \mathrm{~h}$ ( $n=2$ for the latter two time points) per 30 high power fields (Fig. $5 b$ ).

By propidium iodide staining, at $4 \mathrm{wk}$ (peak fibrosis, $3 \mathrm{~d}$ after final $\mathrm{CCl}_{4}$ injection) the mean number of condensed nuclei was $32 \pm 8.3(n=3)$ and remained at a comparable level, a mean of $24 \pm 11(n=3)$ at $3 \mathrm{~d}$ of recovery, before falling to $4.6 \pm 0.58(n=3)$ at $28 \mathrm{~d}$ of recovery (Fig. $5 a)$. Whereas by TUNEL staining at $4 \mathrm{wk}$ (peak fibrosis), the mean number of apoptotic figures was $23 \pm 4.2(n=3)$ per 30 high power fields. The number of TUNEL positive nuclei remained constant for the first $72 \mathrm{~h}$ of recovery before falling to $10.6 \pm 1.5$ per 30 high power fields at $7 \mathrm{~d}$ and reaching $4 \pm 1$ per 30 high power fields at $28 \mathrm{~d}$ of recovery ( $n=3$ at each time point) (Fig. $5 b$ ).

HSC activated by culture on plastic exhibit apoptosis. The in vivo studies suggested that activated HSCs were undergoing apoptosis and demonstrated that TUNEL positive condensed apoptotic bodies were localized to $\alpha$-SMA positive cells. We, therefore, extended our studies to the tissue culture model of HSC activation to determine whether HSC in culture undergo apoptosis. HSCs were activated in primary culture by culture on uncoated tissue culture plastic or after passage. When visualized under phase contrast microscopy (Fig. 6 a), small rounded condensed cells were observed on the surface of the monolayer. These cells could be displaced by agitation of the tissue culture plate, demonstrating that they were very loosely adherent to the monolayer and that some were detached and floating in the culture supernatant. These features are compatible with HSC that have undergone programmed cell death or apoptosis.

To confirm that rounded up cells on the surface of the monolayer were undergoing apoptosis, they were further characterized. By trypan blue exclusion, the cells were demonstrated to be $>99 \%$ viable. When the monolayer was fixed and stained with propidium iodide, small rounded cells with intensely condensed nuclear chromatin, in comparison to the nuclear staining in the monolayer, were obtained in a distribution comparable with the rounded up cells on the monolayer sur-

each specimen by a blinded observer. The results (presented as mean \pm SEM) demonstrate an increase in the number of activated HSC during progressive fibrosis. In spontaneous recovery from fibrosis, there is a 12 -fold reduction in the number of the activated HSCs (a) during the first $7 \mathrm{~d},(b)$ demonstrates a representative example of the $\alpha$-SMA positive cells associated with a fibrotic septae at $4 \mathrm{wk}$ (time 0 ), and (c) is representative of sections stained for $\alpha$-SMA after $28 \mathrm{~d}$ of recovery when few positive cells were observed. 

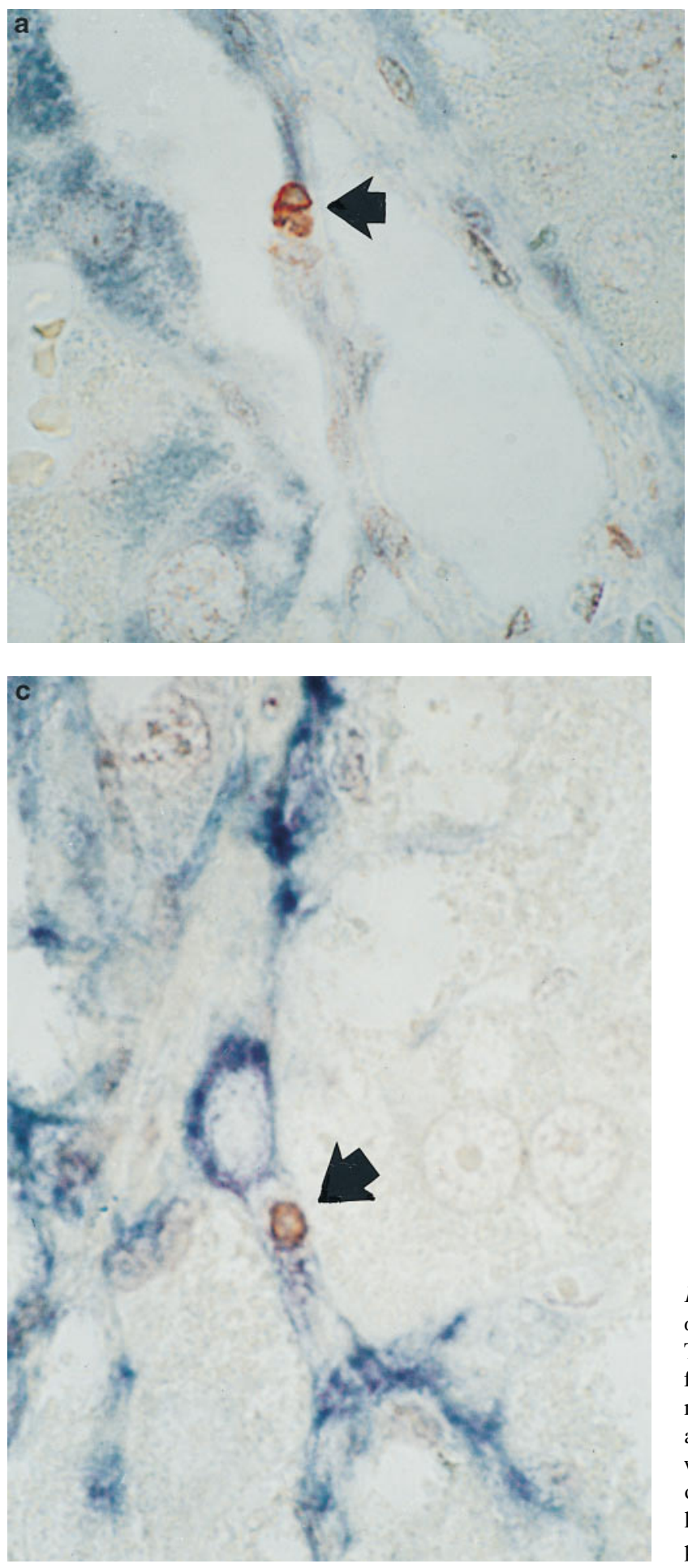

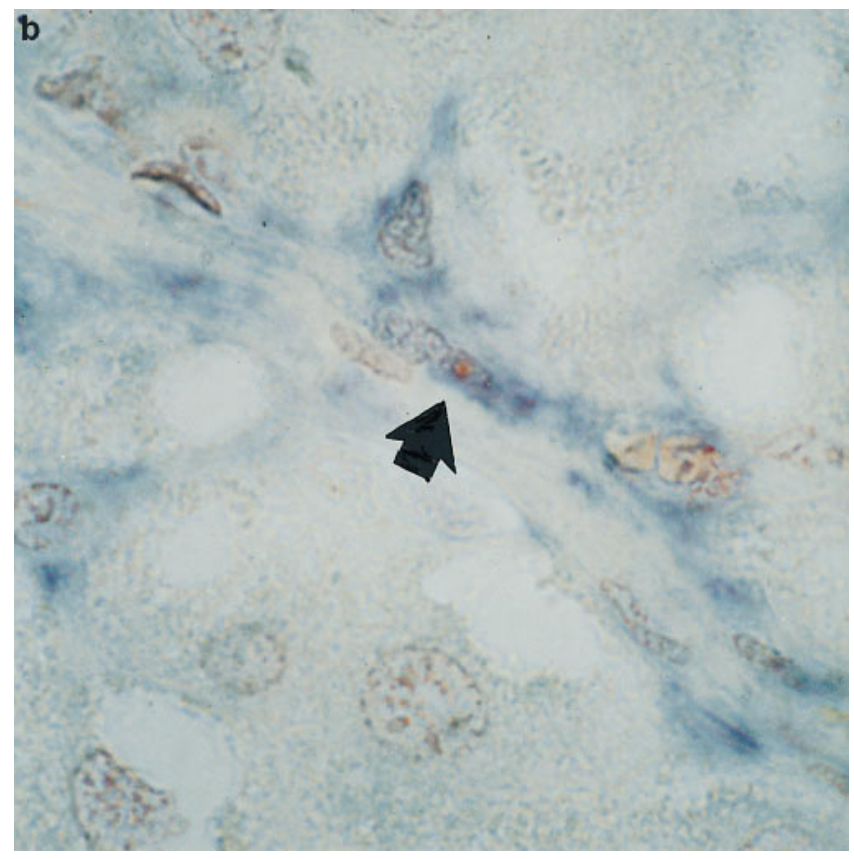

Figure 4. Examples of apoptotic hepatic stellate cells by dual TUNEL and $\alpha$-SMA staining. Liver sections at peak fibrosis were dual stained with TUNEL/ $\alpha$-SMA and apoptotic bodies within activated HSC were identified. $a, b$, and $c$ demonstrate representative examples of TUNEL positive nonparenchymal cell nuclei (red, arrowed) within a fibrotic septum which are $\alpha$-SMA positive (blue), indicating that the TUNEL positive nucleus is within an activated HSC (arrow) $(\times 100)$. Note in $a$, weak $\alpha$-SMA staining of hepatocyte cytoplasm adjacent to the fibrotic septum suggesting that hepatocyte endocytosis of apoptotic stellate cells may have occurred (this pattern was only occasionally observed). face (Fig. $6 b$ ). This finding was confirmed when fixed cells were stained with hematoxylin and eosin and Giemsa (Fig. 6 $c$ ). At higher power, the cellular and nuclear morphology of cells on the monolayer surface and detached cells stained in cytospin preparations of the media demonstrated evidence of cytoplasmic and nuclear blebbing and chromatin condensation (Fig. 6, $d, e$, and f). Furthermore, when these cells were stained using the TUNEL technique, the small, rounded cells on the monolayer surface were found to be strongly positive (Fig. $6 \mathrm{~g}$ ). Those cells with irregular and condensed nuclear morphology demonstrated colocalization of the TUNEL stain, whereas the most condensed nuclei were strongly TUNEL stained (Fig. $6 h$ ).

Activated HSC that had been washed in serum-free media 
a
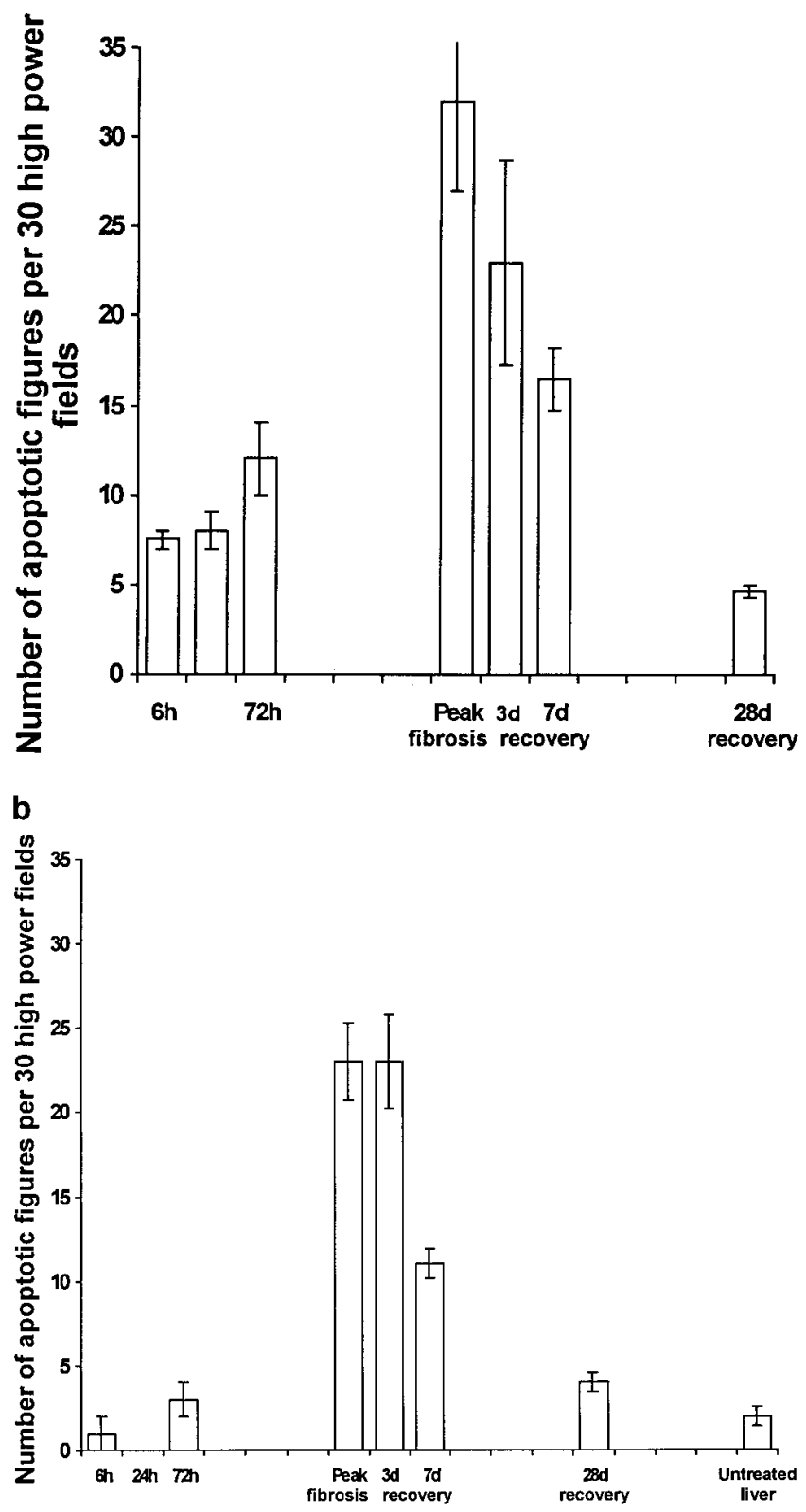

Figure 5. Quantification of nonparenchymal cell apoptotic figures during recovery from $\mathrm{CCl}_{4}$ induced liver fibrosis. Nonparenchymal cell apoptotic figures were quantified in liver sections from the progressive fibrosis model at 6,24 , and $72 \mathrm{~h}$ after $\mathrm{CCl}_{4}$ injection $(n=2$ at each time point); and during the recovery from fibrosis model at $4 \mathrm{wk}$, peak fibrosis ( $3 \mathrm{~d}$ after final $\mathrm{CCl}_{4}$ injection) and at 3,7 , and $28 \mathrm{~d}$ of recovery ( $n=3$ for each time point). Each specimen was stained with propidium iodide ( $a$ ) and TUNEL $(b)$ as described in Methods and the number of nonparenchymal apoptotic figures per 30 high power fields counted as described in Methods. The results are demonstrated graphically (mean \pm SEM, for each time point with each technique) and indicate that nonparenchymal cell (HSC) apoptosis increases in parallel with the numbers of activated HSC during the development of hepatic fibrosis. Moreover, during the first $3 \mathrm{~d}$ of recovery, there is a sustained high level of apoptosis in nonparenchymal cells. and then returned to either serum-containing media (control) or serum-free media alone were stained with acridine orange and counted under fluorescence in the monolayer and supernatant. Incubation of HSC in serum-free conditions for 3 and $6 \mathrm{~h}$ caused an increase in the percentage of cells rounding up, condensing, and detaching into the supernatant, compared with parallel controls (returned to serum). After 3 and $6 \mathrm{~h}$, the corresponding rates of condensation and detachment were $1.8 \%$ (control) versus $8.1 \%$ (serum free) and $1.9 \%$ (control) and $8.9 \%$ (serum free), respectively (data representative of three independent experiments with passaged cells and six experiments with primary cultures of cells). Cell loss from the monolayer was observed at a similar rate for up to $120 \mathrm{~h}$ of culture in serum-free conditions $1 \%$ (control) versus $11 \%$ (serum free) (representative of three independent experiments with passaged cells).

Expression of procollagen-I, TIMP-1, TIMP-2, and MMP13 during spontaneous recovery from fibrosis. To examine possible mechanisms of resolution of fibrosis during recovery, these studies were complemented by analysis of procollagen-1, TIMP-1, TIMP-2, and MMP-13 mRNA content in liver homogenates. After $28 \mathrm{~d}$ of recovery, procollagen-I mRNA expression had fallen significantly to $9 \%$ of the level observed at peak fibrosis (Figs. 7 and $8 d$ ) close to the level observed in untreated, normal liver ( $6 \%$ of the value observed at peak fibrosis). Levels of TIMP-1 mRNA were observed to decrease rapidly over the first $7 \mathrm{~d}$ of recovery. TIMP-1 mRNA expression fell to $28 \%$ of the level observed at peak fibrosis by $7 \mathrm{~d}$, a value close to that observed in normal liver $(26 \%$ of the level observed at peak fibrosis) (Figs. 7 and $8 a$ ). TIMP-2 mRNA expression demonstrated a similar pattern during recovery. By day 7, TIMP-2 mRNA levels were $30 \%$ of the value observed at peak fibrosis (Figs. 7 and $8 b$ ), and below the level observed in untreated control liver $(67 \%$ of the value observed at peak fibrosis). In contrast to the pattern of expression of TIMP-1 and TIMP-2, expression of interstitial collagenase (MMP-13) did not significantly change from the level observed at peak fibrosis. Interstitial collagenase (MMP-13) expression never fell below $94 \%$ of the peak fibrosis level throughout the recovery model (Figs. 7 and $8 c$ ) and remained raised above levels observed in normal liver ( $22 \%$ of the value of peak fibrosis).

Collagenase activity in liver homogenates increases with recovery from fibrosis. To further examine mechanisms responsible for degradation of fibrotic matrix during recovery, interstitial collagenase activity was examined. When liver homogenates from time $0,3,7$, and $14 \mathrm{~d}$ of recovery from fibrosis were assayed overall interstitial collagenase activity was found to increase from 5,155 through 7,$767 ; 8,996$ to $9,514 \mathrm{cpm} / \mathrm{mg}$ protein, respectively, for each time point. Collagenase activity in normal liver was found to be close to that observed at $14 \mathrm{~d}$ being 9,219 cpm/mg protein. (Data expressed as mean for duplicate sample analysis of two livers at each time point.)

\section{Discussion}

The results presented in this report demonstrate that even relatively advanced fibrosis in rat liver can undergo remodeling with restoration of the normal hepatic architecture over a comparatively short time course. Our studies suggest that the key event in this process is the loss of activated stellate cells mediated by apoptosis. The process of matrix degradation may be facilitated by the diminution of activated HSCs that have been 

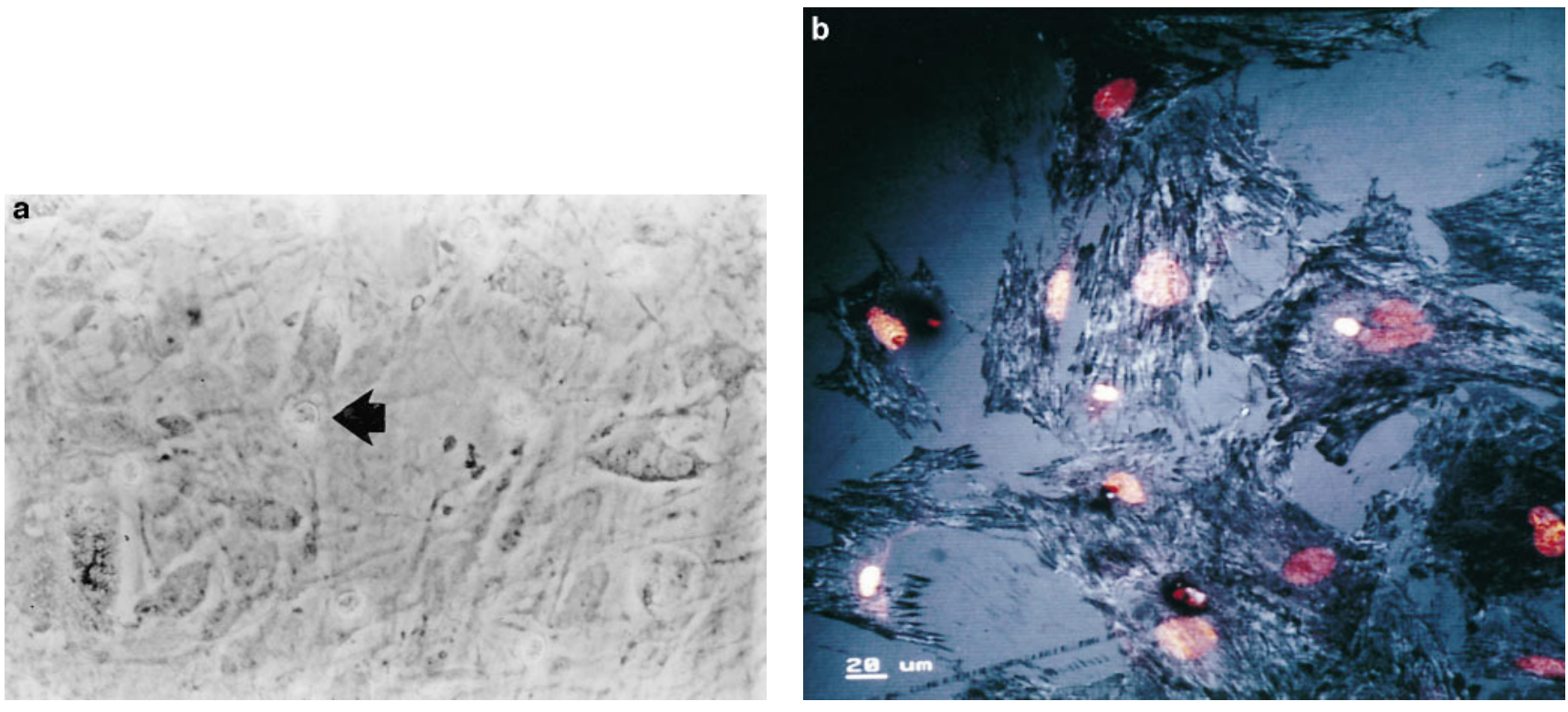
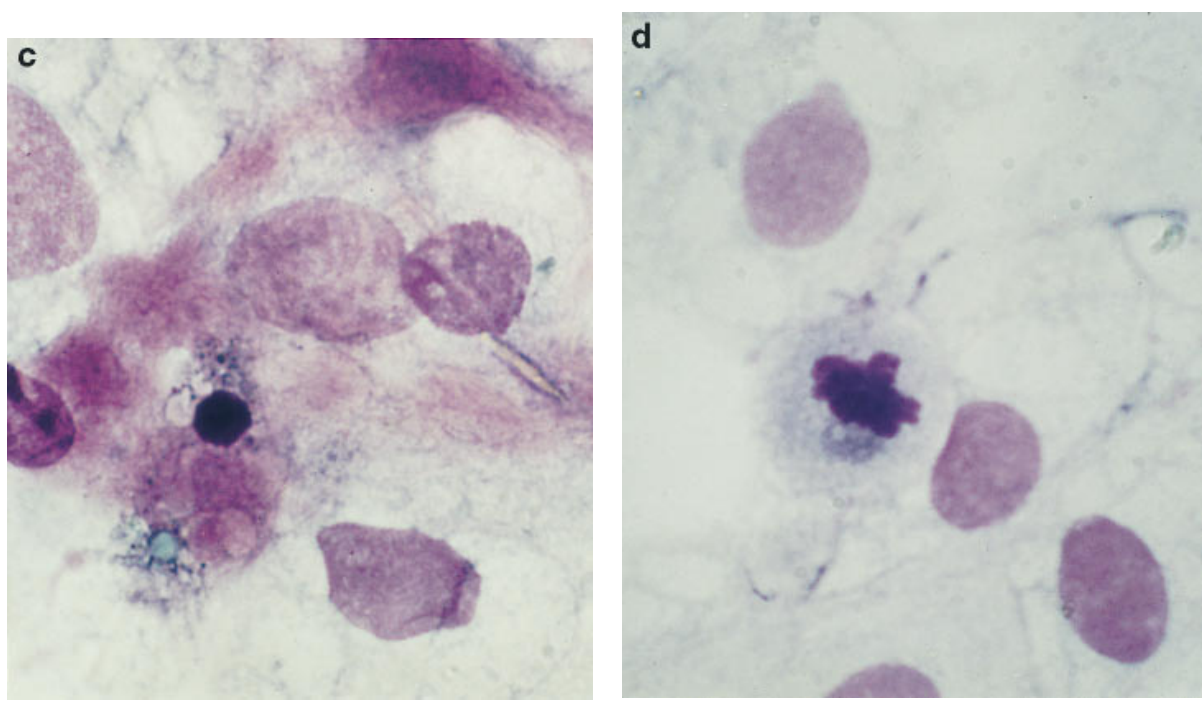

f

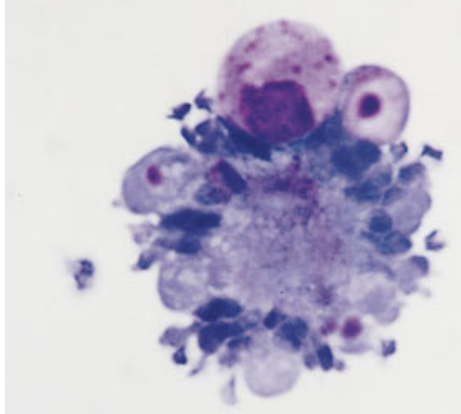

h

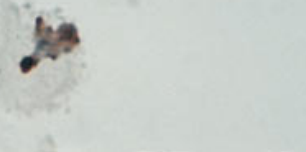

e

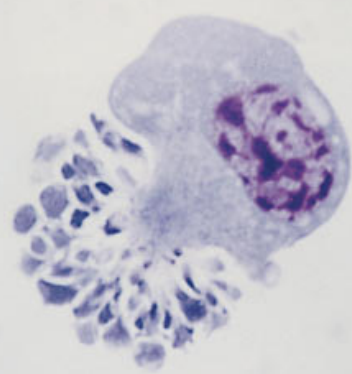

Figure 6. Evidence of apoptosis in HSCs activated by culture on plastic. Passaged activated HSC cultured in serum-free media for $4 \mathrm{~h}$ were studied for evidence of apoptosis. Under phase contrast, cells on the surface of the monolayer could be demonstrated to be rounded up and condensed (arrowed) $(a)(\times 25)$. Fixed cells were stained with propidium iodide and photographed by confocal microscopy. The composite image presented in $b$ is composed of the cell morphology photographed under tungsten light, with the propidium iodide-stained nuclei photographed under fluorescent light superimposed. The intensely condensed nuclei of the rounded cells on the surface fluoresce very strongly (bright white) and contrast in size and intensity with the normal cell nuclei fluorescing red in the monolayer $(\times 25)$. Giemsa stains of activated HSC after $4 \mathrm{~h}$ in serum-free media in the fixed monolayer and cytospin preparations of detached HSC in the media supernatant demonstrated clear evidence of chromatin condensation and nuclear and cytoplasmic blebbing $(c, d, e$, and $f)(\times 100)$. By TUNEL stain the most condensed cells demonstrated evidence of DNA fragmentation and were strongly staining (arrowed) (examples in $g$ and $h, \times 25$ and $\times 40$, respectively). 


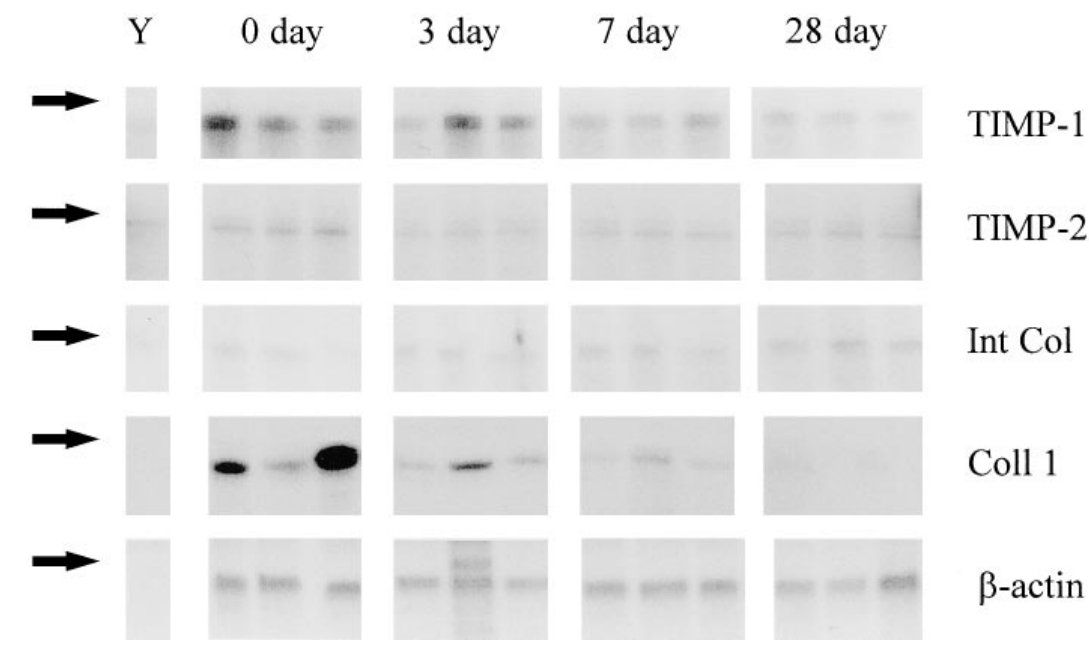

Figure 7. Ribonuclease protection assays for TIMP-1, TIMP-2, interstitial collagenase (MMP-1/ MMP-13) and procollagen-I during spontaneous recovery from $\mathrm{CCl}_{4}$ induced fibrosis. Ribonuclease protection assays for TIMP-1, TIMP-2, interstitial collagenase (MMP-1/MMP-13), procollagen-1, and $\beta$-actin were performed as described in Methods. Arrows indicate position of probes, $\mathrm{Y}=$ negative control yeast RNA. Samples are grouped at each time point as follows: $0 \mathrm{~d}-4 \mathrm{wk}$ (peak fibrosis) (samples i, ii, iii), and $3 \mathrm{~d}$ (samples i, ii, iii), $7 \mathrm{~d}$ (samples i, ii, iii), and $28 \mathrm{~d}$ (samples i, ii, iii) of spontaneous recovery from fibrosis. Each lane represents the result from an individual separate liver from a rat killed at that time point of the model. shown to express procollagen-I, TIMP-1, and TIMP-2. Such a pattern of gene expression in the presence of unaltered rat interstitial collagenase expression may lead to an increase in overall collagenase activity and the degradation of existing fibrotic matrix.

In this study, we have confirmed previous reports that progressive fibrosis is associated with an increase in desmin and $\alpha$-SMA positive cells. We have additionally shown that this change is associated with a parallel increase in the rate of nonparenchymal cell apoptosis. Moreover, spontaneous recovery from liver fibrosis is accompanied by loss of $\alpha$-SMA positive activated HSCs, and we have presented evidence that this cell loss is mediated by apoptosis. The net effect on HSC numbers is likely to be enhanced by a reduction in HSC proliferation consequent to withdrawal of the fibrogenic stimulus. The reduction in desmin positive cells (quiescent and activated HSCs) observed during recovery, suggests that loss of activated HSCs by programmed cell death is occurring as opposed to a reversion of phenotype from the activated to a quiescent one (31). We cannot exclude the possibility that some cells undergo reversion from an activated to a quiescent phenotype, but our data suggest that the majority of loss of $\alpha$-SMA positive cells is mediated via apoptosis. The observed disparity in desmin and $\alpha$-SMA positive cell numbers during the model may be related to differing antibody specificities and/or the failure of all activated HSC to express desmin. Our data, however, suggest that apoptosis is a major factor regulating HSC numbers during the injury-fibrosis-recovery sequence.

We have used a series of complementary techniques in vivo, in tissue sections, and in cell culture to demonstrate HSC apoptosis and have adopted strict morphological criteria to define the likely lineage of apoptotic figures identified in liver sections. This approach has previously been applied successfully to studies of mesangial cell apoptosis in renal injury (26). To this end, during the recovery model we have only quantified apoptotic figures that have been observed in clear association with fibrotic septae or the residues of fibrotic septae. By immunostaining, we have demonstrated that the cells in these areas are almost exclusively $\alpha$-SMA positive and by dual staining we have demonstrated that TUNEL positive condensed nuclei within the fibrotic bands are observed within $\alpha$-SMA positive cell, indicating that any apoptosis observed in these same regions is occurring in activated HSCs. Moreover, the diminution in nonparenchymal cells within and adjacent to the septae is not associated with evidence of necrosis or inflammation, further supporting the hypothesis that, in these cells, death is programmed. Propidium iodide was used to identify and quantify condensed nuclei, and TUNEL staining used to identify and quantify cells with oligonucleosomal DNA fragmentation typical of apoptosis. These techniques confirmed that cells with a distribution consistent with activated HSCs were undergoing apoptosis. Used together to obtain an overview of the chronology of the development and recovery from fibrosis, the TUNEL and propidium iodide methods demonstrated that nonparenchymal cell apoptosis accompanies the increase in $\alpha$-SMA positive cell numbers, which occur during experimental fibrosis (Figs. $3 a$ and 5, $a$ and $b$ ). A recent study by De Bleser et al. (32) noted as an incidental finding that TUNEL positive nuclei in fibronectin positive cells (activated HSC) within and adjacent to fibrotic septae were present after chronic $\mathrm{CCl}_{4}$ intoxication, thus confirming our observations using an alternative HSC marker. The first $7 \mathrm{~d}$ of recovery is then associated with a period of sustained apoptotic activity in nonparenchymal cells, in a distribution consistent with activated HSC. The rate of apoptosis then drops to a lower level at $28 \mathrm{~d}$ of recovery. During the first $3 \mathrm{~d}$, the number of $\alpha$-SMA positive cells is halved and by $7 \mathrm{~d}$ is reduced by a factor of 12 compared with peak fibrosis. Saile et al. (33) have recently described $\mathrm{HSC}$ apoptosis in vivo after a single injection of $\mathrm{CCl}_{4}$ and have gone on to demonstrate that activated HSCs express Fas (APOI/CD95) and undergo apoptosis in response to Fas ligand. Taken together with our work, these data provide strong evidence that HSC cell numbers are controlled by apoptosis in addition to proliferation during progressive fibrosis and particularly during recovery from fibrosis. The finding that the absolute numbers of apoptotic cells at peak fibrosis and $3 \mathrm{~d}$ of recovery represents a tiny portion of the overall number of $\alpha$-SMA positive cells, does not diminish the importance of apoptosis as a mechanism for cell loss. Indeed, the process of apoptosis is metachronous within a population of cells and, therefore, at each time point only a snapshot of the apoptotic activity has been determined. Moreover, in the pres- 


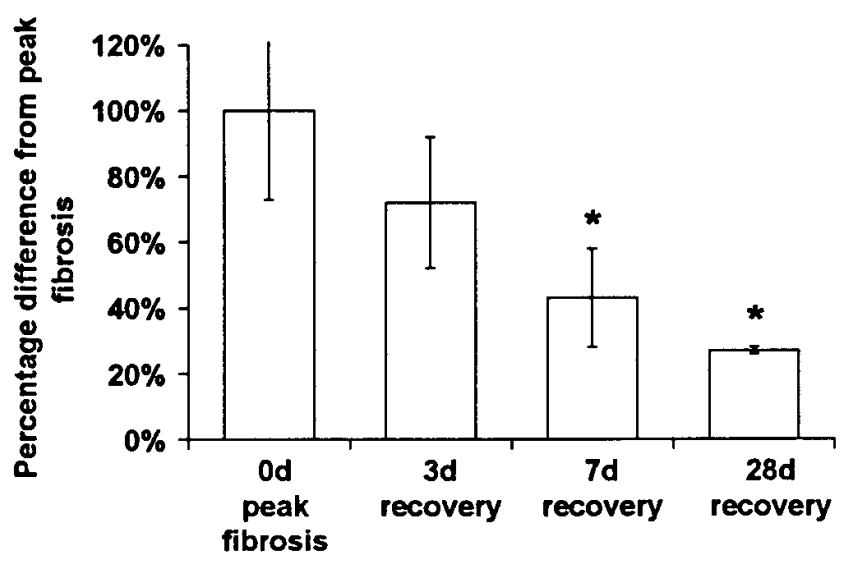

C

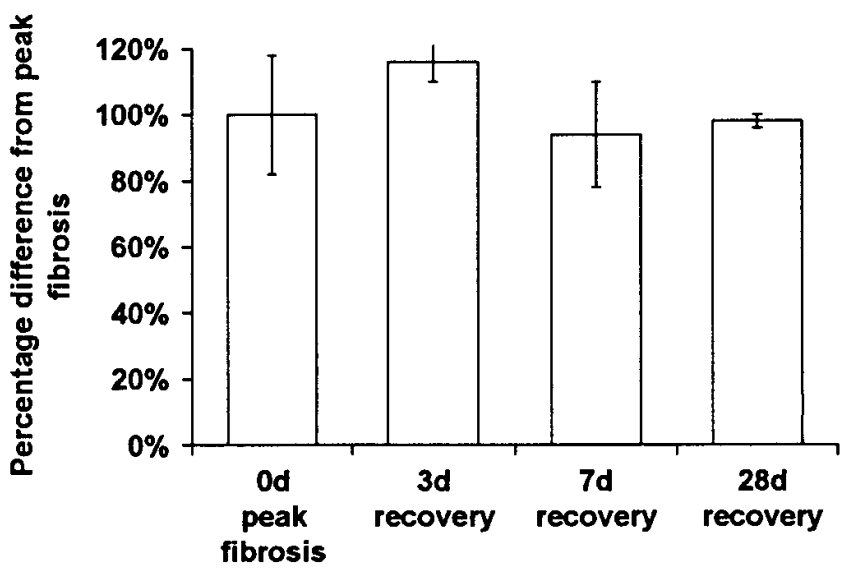

b

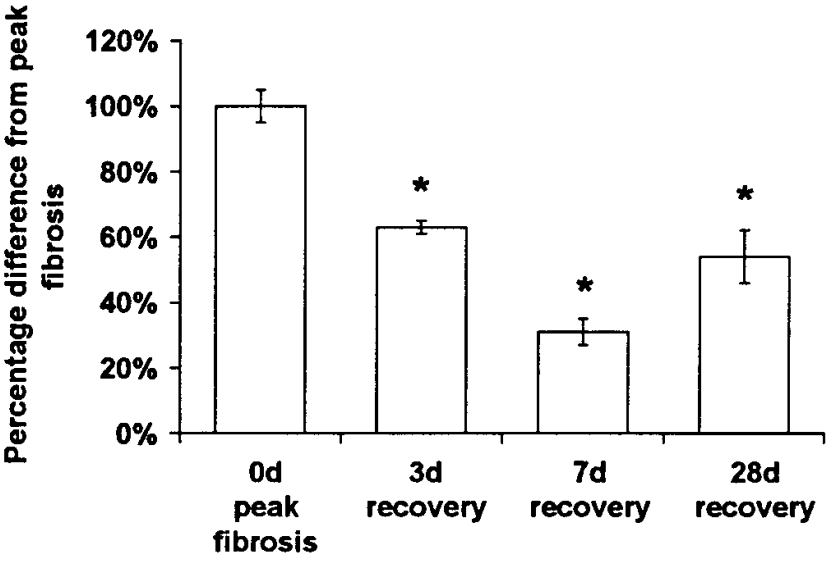

d

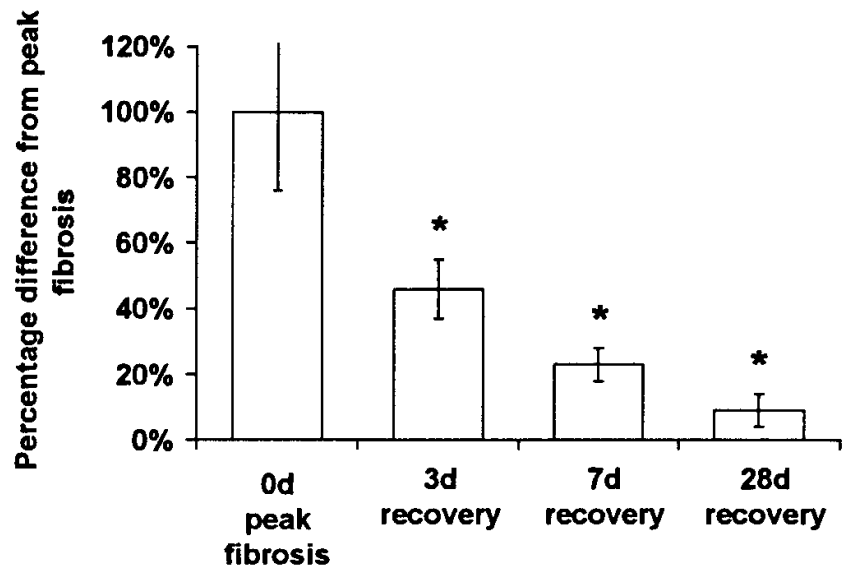

Figure 8. Densitometric analysis of expression of mRNA for TIMP-1, TIMP-2, interstitial collagenase (MMP-1/MMP-13), and procollagen-I during spontaneous recovery from $\mathrm{CCl}_{4}$ induced fibrosis. Densitometric analysis of mean TIMP-1 (a), TIMP-2 (b), interstitial collagenase (MMP-1/ MMP-13) (c), and procollagen-1 $(d)$ expression at each time point during recovery is plotted. Values are expressed as percentage change from: $0 \mathrm{~d}-4 \mathrm{wk}$ (peak fibrosis), which has been given the arbitrary value of $100 \%$. Data presented represent the mean for each group of three livers at each time point $(* 0.01<P<0.05$ in comparison to the value observed at peak fibrosis by Mann-Whitney test).

ence of a constant rate of cellular proliferation, comparatively minor changes in the rate of apoptosis may result in dramatic clearance of cells (26).

We have used highly pure cultures of activated stellate cells to confirm that activated HSCs undergo apoptosis in cell culture. The model of activation by culture on plastic in the presence of serum has been demonstrated to recapitulate many of the features of activation observed during injury in vivo, including expression of $\alpha$-SMA, secretion of collagen I, expression of TIMP-1 and -2 and proliferation $(1,10,15,19)$. Our results indicate that apoptosis occurs in activated HSCs in this model (Fig. 6, $a-i$ ). As in our in vivo studies, we have used a series of complementary methods to demonstrate apoptosis in HSC culture and have demonstrated remarkably consistent results. First, we observed that, in populations of both primary culture and passaged HSCs, cells on the surface of the monolayer round up and detach in a manner identical to that described for apoptotic fibroblasts in other models $(34,35)$. On the basis of trypan blue exclusion these cells are viable and have condensed nuclei by acridine orange and propidium iodide staining (Fig. 6 b). Moreover, individual nuclei, viewed under high power, demonstrated chromatin condensation, blebbing, and fragmentation (Fig. 6, $b-f$ ), and oligonucleosomal DNA fragmentation was confirmed by in situ TUNEL staining (Fig. 6, $g$ and $i$ ). All of these features are characteristic of apoptosis and distinguish programmed cell death from necrosis. We have further demonstrated that serum deprivation of activated HSCs has the result of increasing the proportion of activated HSCs condensing and detaching from the monolayer, indicating that exposure of HSC to growth factors actively prevents entry into the apoptotic process in addition to promoting proliferation.

Loss of activated HSCs alone cannot explain the resolution of fibrosis and, in particular, the progressive loss of the preexisting fibrotic matrix over $28 \mathrm{~d}$, nor the rapid degradation of matrix collagen fibrils over the first $7 \mathrm{~d}$ of recovery. Although the results of the first part of the study provide a possible explanation for the diminution in procollagen-I mRNA based on 
HSC apoptosis, the question of the mechanism regulating matrix degradation needed further clarification. We, therefore, quantified expression of rat interstitial collagens (MMP-13) in parallel with two collagenase inhibitors TIMP-1 and -2 during recovery from liver fibrosis. Our results indicate that the extensive remodeling is temporally correlated with a diminution of TIMP-1 and TIMP-2 expression. In contrast, rat interstitial collagenase (MMP-13) mRNA expression remained at levels comparable to peak fibrosis and does not diminish in parallel with TIMP-1 and -2 through recovery. These data suggested that an important factor promoting matrix remodeling is the removal of the inhibitory influence of the TIMPs on collagenase activity. As we have previously shown that TIMP-1 and -2 are produced by activated HSC, we further suggest that the hepatic reduction in TIMP-1 and - 2 mRNA may be, at least in part, a consequence of the reduction in numbers of activated HSCs although a reduction in TIMP mRNA expression by HSCs cannot be excluded as an alternative or concurrent mechanism. We went on to analyze collagenase activities in whole liver supernatants during recovery from fibrosis and observed that relative to normal liver overall activity is decreased at peak fibrosis and increases during the first 14-d of recovery, coinciding with the diminution of TIMP-1 and -2 mRNAs. This data suggests that collagenase activity is constitutive and may play a part in the matrix turnover of normal liver, but becomes inhibited by TIMPs during fibrogenesis.

Our findings in the recovery model further support the importance of TIMPs in regulating collagen degradation in liver and complement our previous studies of interstitial collagenase (MMP-1/MMP-13) and TIMPs in fibrotic human liver and experimental rat liver fibrosis $(10,15,21)$. These observations are supported by data presented by other groups, which indicate that collagenase activities detected in tissue homogenates initially rise with fibrotic injury but then fall with progression of fibrosis to cirrhosis $(17,18,36-38)$ and that fibrotic human liver disease is associated with raised serum TIMP levels, which are inversely correlated to collagenase activity (2224). Moreover, serum TIMP levels in patients with liver fibrosis are inversely correlated to collagenase activity, which can be unmasked by removal of the bound TIMP (22-24).

Our data have highlighted key areas in the process of spontaneous recovery from relatively advanced liver fibrosis. First, the process of HSC apoptosis has been demonstrated to be central to the diminution of activated HSC numbers during recovery. Second, this study, in combination with our previous work $(10,11,13,15,19,21)$, provides data to suggest that alterations in matrix degradation may be important, not only to the progression of liver fibrosis, but also to its natural resolution in this model of liver injury. This has important implications for the future development of therapeutic antifibrotic strategies in the liver.

\section{Acknowledgments}

The authors gratefully acknowledge the support of the British Liver Trust and the Wessex Medical Trust and the extremely helpful advice of B. Wilkins and C. Meehan (Department of University Pathology, Southampton General Hospital, Hampshire, UK).

J.P. Iredale is a Medical Research Council (MRC) Clinician Scientist and C. Hovell is an MRC Training Fellow. At the time of these studies, M.J.P. Arthur was the recipient of a Wellcome Trust project grant (038712/Z/93/A/1.5), which supported R.C. Benyon.

\section{References}

1. Friedman, S.L. 1993. The cellular basis of hepatic fibrosis: mechanisms and treatment strategies. N. Engl. J. Med. 328:1828-1835.

2. Rojkind, M., and M.A. Dunn. 1979. Hepatic fibrosis. Gastroenterology. 76:849-863.

3. Hunt, J. 1992. Long-term follow-up of patients with hepatitis B treated with interferon. Interferons Cytokines. 20:6-9.

4. Abdel-Aziz, G., G. Lebeau, P.Y. Rescan, B. Clement, M. Rissel, Y. Deugnier, J.P. Campion, and A. Guillouzo. 1990. Reversibility of hepatic fibrosis in experimentally induced cholestasis in rat. Am. J. Pathol. 137:1333-1342.

5. Vaux, D.L., and A. Strasser. 1996. The molecular biology of apoptosis. Proc. Natl. Acad. Sci. USA. 93:2239-2244.

6. Evan, G.I., L. Brown, M. Whyte, and E. Harrington. 1995. Apoptosis and the cell cycle. Curr. Opin. Cell Biol. 7:825-834.

7. Patel, T., and G.J. Gores. 1995. Apoptosis and hepatobiliary disease. Hepatology. 21:1725-1741.

8. Fraser, A., and G. Evan. 1996. A license to kill. Cell. 85:781-784.

9. Freije, J.M.P., I. Diezitza, M. Balbin, L.M. Sanchez, R. Blasco, J. Tolivia, and C. Lopezotin. 1994. Molecular cloning and expression of collagenase-3, a novel human matrix metalloproteinase produced by breast carcinomas. J. Biol. Chem. 269:16766-16773.

10. Benyon, R.C., J.P. Iredale, S. Goddard, P.J. Winwood, and M.J.P. Arthur. 1996. Expression of tissue inhibitor of metalloproteinases-1 and -2 is increased in fibrotic human liver. Gastroenterology. 110:821-831.

11. Iredale, J.P., P.J. Winwood, C.A. Kawser, and M.J.P. Arthur. 1993. Lipocyte proliferation in the $C$. parvum model of macrophage-induced liver injury is associated with expression of 72-kD type IV collagenase/gelatinase. In Cells of the Hepatic Sinusoid, Vol. 4. D.L. Knook and E. Wisse, editors. Kupffer Cell Foundation, Leiden. 105-108.

12. Herbst, H., O. Heinrichs, D. Schuppan, S. Milani, and H. Stein. 1991. Temporal and spatial patterns of transin/stromelysin RNA expression following toxic injury in rat liver. Virchows Arch. B Cell Pathol. 60:295-300.

13. Arthur, M.J.P. 1994. Matrix degradation in the liver. In Fat-Storing Cells and Liver Fibrosis. C. Surrenti, A. Casini, S. Milani, and M. Pinzani, editors. Kluwer Academic Publishers, Dordrecht, Germany. 110-127.

14. Li, J.J., C.I. Kim, M.A. Leo, K.M. Mak, M. Rojkind, and C.S. Lieber. 1992. Polyunsaturated lecithin prevents acetaldehyde-mediated hepatic collagen accumulation by stimulating collagenase activity in cultured lipocytes. Hepatology. 15:373-381.

15. Iredale, J.P., R.C. Benyon, M.J.P. Arthur, W.F. Ferris, R. Alcolado, P.J. Winwood, N. Clark, and G. Murphy. 1996. Tissue inhibitor of metalloproteinase-1 messenger RNA expression is enhanced relative to interstitial collagenase messenger RNA in experimental liver injury and fibrosis. Hepatology. 24:176-184.

16. Okazaki, I., and K. Maruyama. 1974. Collagenase activity in experimental hepatic fibrosis. Nature. 252:49-50.

17. Perez-Tamayo, R., I. Montfort, and E. Gonzalez. 1987. Collagenolytic activity in experimental cirrhosis of the liver. Exp. Mol. Pathol. 47:300-308.

18. Maruyama, K., L. Feinman, Z. Fainsilber, M. Nakano, I. Okazaki, and C.S. Lieber. 1982. Mammalian collagenase increases in early alcoholic liver disease and decreases with cirrhosis. Life Sci. 30:1379-1384.

19. Iredale, J.P., G. Murphy, R.M. Hembry, S.L. Friedman, and M.J.P. Arthur. 1992. Human hepatic lipocytes synthesize tissue inhibitor of metalloproteinases-1 (TIMP-1): implications for regulation of matrix degradation in liver. J. Clin. Invest. 90:282-287.

20. Hovell, C.J., R.C. Benyon, S.J. Pawley, J.P. Iredale, and M.J.P. Arthur 1997. Membrane-type matrix metalloproteinase (MTMMP) is upregulated in liver fibrosis. J. Hepatol. 26:133.

21. Iredale, J.P., S. Goddard, G. Murphy, R.C. Benyon, and M.J.P. Arthur 1995. Tissue inhibitor of metalloproteinase-1 and interstitial collagenase expression in autoimmune chronic active hepatitis and activated human hepatic lipocytes. Clin. Sci. 89:75-81.

22. Murawaki, Y., H. Yamamoto, H. Kawasaki, and H. Shima. 1993. Serum tissue inhibitor of metalloproteinases in patients with chronic liver disease and with hepatocellular carcinoma. Clin. Chim. Acta. 218:47-58.

23. Muzzillo, D.A., M. Imoto, Y. Fukuda, Y. Koyama, S. Saga, Y. Nagai, and T. Hayakawa. 1993. Clinical evaluation of serum tissue inhibitor of metalloproteinases-1 levels in patients with liver diseases. J. Gastroenterol. Hepatol. 8: 437-441.

24. Murawaki, Y., H. Kawasaki, and H. Burkhardt. 1994. Serum collagenase activity in chronic liver diseases. Pathol. Res. Pract. 190:929-933.

25. Rockey, D.C., J.K. Boyles, G. Gabbiani, and S.L. Friedman. 1992. Rat hepatic lipocytes express smooth muscle actin upon activation in vivo and in culture. J. Submicrosc. Cytol. Pathol. 24:193-203.

26. Baker, A.J., A. Mooney, J. Hughes, D. Lombardi, R.J. Johnson, and J. Savill. 1994. Mesangial cell apoptosis: the major mechanism for resolution of glomerular hypercellularity in experimental mesangial proliferative nephritis. $J$. Clin. Invest. 94:2105-2116.

27. Gavrieli, Y., Y. Sherman, and S.A. Ben-Sasson. 1992. Identification of programmed cell death via specific labeling of nuclear DNA fragmentation. $J$. Cell Biol. 119:493-501.

28. Arthur, M.J.P., S.L. Friedman, F.J. Roll, and D.M. Bissell. 1989. Lipo- 
cytes from normal rat liver release a neutral metalloproteinase that degrades basement membrane (type IV) collagen. J. Clin. Invest. 84:1076-1085.

29. Bergman, I., and R. Loxley. 1963. Two improved and simplified methods for the spectrophotometric determination of hydroxyproline. Anal. Chem. 35:1961-1965.

30. Chomczynski, P., and N. Sacchi. 1987. Single-step method of RNA isolation by acid guanidinium thiocyanate-phenol-chloroform extraction. Anal. Biochem. 162:156-159.

31. Burt, A.D. 1993. Cellular and molecular aspects of hepatic fibrosis. $J$. Pathol. 170:105-114.

32. De Bleser, P.J., T. Niki, T. Xu, V. Rogiers, and A. Geerts. 1997. Localization and cellular sources of activins in normal and fibrotic rat liver. Hepatology. 26:905-912.

33. Saile, B., T. Knittel, N. Matthes, P. Schott, and G. Ramadori. 1997. CD95/CD95L-mediated apoptosis of the hepatic stellate cell. Am. J. Pathol.
151:1265-1272.

34. Fanidi, A., E.A. Harrington, and G.I. Evan. 1992. Cooperative interaction between c-myc and bcl-2 proto-oncogenes. Nature. 359:554-556.

35. Evan, G.I., A.H. Wyllie, C.S. Gilbert, T.D. Littlewood, H. Land, M. Brooks, C.M. Waters, L.Z. Penn, and D.C. Hancock. 1992. Induction of apoptosis in fibroblasts by c-myc protein. Cell. 69:119-128.

36. Carter, E.A., M.J. McCarron, E. Alpert, and K.J. Isselbacher. 1982. Lysyl oxidase and collagenase in experimental acute and chronic liver injury. Gastroenterology. 82:526-534.

37. Murawaki, Y., S. Yamada, M. Koda, and C. Hirayama. 1990. Collagenase and collagenolytic cathepsin in normal and fibrotic rat liver. J. Biochem. 108:241-244.

38. Montfort, I., R. Perez-Tamayo, A.M. Alvizouri, and E. Tello. 1990. Collagenase of hepatocytes and sinusoidal liver cells in the reversibility of experimental cirrhosis of the liver. Virchows Arch. B Cell Pathol. 59:281-289. 